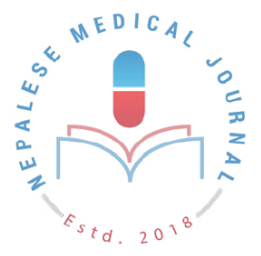

\title{
Audiology and Speech-Language Pathology Services in Pediatric Population During COVID Pandemic in Nepal
}

\author{
Prabuddha Bhatarai ${ }^{1}$,Biraj Bhattarai ${ }^{1}$, Sureshwor Lal Karna ${ }^{2}$ \\ ${ }^{1}$ All India Institute of Speech and Hearing, Mysore, Karnataka, India \\ ${ }^{2}$ Tribhuvan University Teaching Hospital, Maharajgunj, Kathmandu, Nepal
}

\section{ABSTRACT}

\begin{abstract}
The pandemic has affected the daily working of people worldwide, including Audiology and speechlanguage services. The Audiology and speech-language pathology services both require proximity to the patients and hence increases the risk for transmission of the virus. These services are required even during this time are immense to utilize a critical period of language development. Teleservices are a suitable option to assess and rehabilitate the client at this time. In a country like Nepal, where limited professionals are working in this field, telehealth not only reduces transmission risk but also could be useful for patients who cannot attend clinics regularly due to the unavailability of services near their reach. The Speech and Hearing Association and all professionals working in this field should be ready to accept the changes that will eventually lead to the growth of the field and the reach of the services for the patients in need.
\end{abstract}

Keywords: Audiology; COVI-19; Speech language; Telepractice
Correspondence:

Prabuddha Bhatarai

All India Institute of Speech and Hearing, Mysore, Karnataka, India

ORCID ID: 0000-0002-9866-4491

Email:prabuddha404@gmail.com

Submitted: 10th October 2020

Accepted: 7th December 2020

Source of Support: None

Conflict of Interest: None

Citation: Bhatarai P, Bhattarai B, Karna SL. Audiology and Speech-Language Pathology Services in Pediatric Population during COVID pandemic in Nepal.NMJ 2020;3(2):398-401. DOI 10.3126/nmj.v3i2.32325

\section{INTRODUCTION}

The outbreak in Wuhan of China with unknown origin and named as acute severe acute respiratory syndrome coronavirus 2 (SARSCoV-2) or coronavirus disease 2019 (COVID-19), a pandemic has struck the world affecting overall everyday human life. ${ }^{1,2}$ It has the symptoms of fever, cough, sore throat, breathlessness, fatigue, malaise initially and can progress to pneumonia, Acute Respiratory Distress Syndrome (ARDS), and multi-organ dysfunction, which can be fatal in the final stage in some severe cases. $^{3}$ The first case reported outside China was in Thailand on $13^{\text {th }}$ January 2020, and it rapidly spread over Asian countries like Japan. Republic of South Korea and Singapore. Subsequently, it was announced as a global emergency by the World Health Organization (WHO) on 30th January $2020 .^{2}$
Meanwhile, Nepal could not withstand the global pandemic, and hence on $23^{\text {rd }}$ January 2020, the first case of COVID-19 was reported. Nepal government implemented a lockdown strictly from $24^{\text {th }}$ March to minimize the outbreak (MoHP, Nepal). The central and local government had to shut down most of the occupation and service providing centers due to the outbreak. Along with people's daily living, the country's economy has been shattered badly by this, but individuals with disabilities encounter the worst. ${ }^{4}$

Apart from the emergency services, even the health service providers were not functional during the time of the pandemic. Most Audiological procedures require proximity with patients, for example (giving instructions for testing, placement of headphones, 
probes, electrodes, hearing aid fitting, etc.) as well as during counseling. Similarly, speech-language services require face-toface communications, eye contact, and communicative play with children with language and behavioral issues. Audiologist and Speech therapist being non-functional has affected individuals with hearing and other speech impairments. Early diagnosis and early intervention are critical to restoring the near-normal speech and hearing in those individuals. Neonatal hearing screening is essential for early identification, and the delay in diagnosis after three years has shown to have a significant impact on a child's life. Better communication, social, behavioral, and intelligence skills are possible if they get intervention before six months. It is crucial to utilize the child's critical period for language development, five years or earlier, to provide enough benefit. With the nonavailability of both speech and hearing professionals, it has been challenging to carry out newborn screening in hospitals, which further delays the intervention.

\section{COVID-19 and Audiology Services}

Audiological testing and rehabilitation had both been affected during the nationwide lockdown. Even with the removal of lockdown, the primary services being located at the Kathmandu valley, limited transportation, and not all centers being functional have made it difficult for patients to avail themselves. Sudden Sensorineural Hearing loss (SNHL) is also an otological emergency that requires immediate medical attention. Delay in more than 14 days(two weeks) for treatment of SNHL can significantly impact an individual's mode of communication and deteriorate the overall quality of life. ${ }^{5}$ Cochlear implant surgeries are also canceled due to pandemics. ${ }^{6}$ Regular Mapping of Cochlear implants will be unavailable for children recently undergone surgery. Even with those patients using hearing aids, it is difficult to come for reprogramming, retesting, and continuing the centerbased therapy. In the case of device failure, professionals are unavailable for troubleshooting and repair, which will prevent them from hearing speech sounds until professionals are available. This may hinder the early rehabilitation process for the children and cause auditory deprivation.

\section{COVID-19 and Speech-Language Services}

There is a negative effect on the service delivery of SLP(SpeechLanguage Pathology)due to the novel Coronavirus. The risk of transmission is very high if precaution is not taken. As SpeechLanguage Pathology services require close contact with both parents and children with disabilities, the chance of transmission is even more. During assessment and intervention as an SLP, it is crucial to maintain face-to-face communication with their clients to visualize articulators' movement, and good eye contact is preliminary. Due to this fact, SLP cannot mask their face and articulators with Personal Protective Equipment(PPE). Moreover, SLPs have to frequently use toys for therapies and sometimes even touch the child during the session, severely increasing the chances of spread. Therefore, it is almost impossible for SLPs to provide service in-person to individuals with disabilities during the CoVID-19 outbreak. ${ }^{7}$

\section{Psychological Impact on Families}

Parents of differently-abled individuals and communication disabilities (e.g., hearing impairment, autism, spoken language disorders, Fluency Disorders, Intellectual disabilities.) face recurrent issues like stress, anxiety, and negative feelings. ${ }^{7-10}$ The pandemic of COVID has brought various problems in the parents' daily living, one major being the financial crisis. This issue of finance and insufficient income due to lockdown develops family disputes. ${ }^{11}$ Meanwhile, this harms quality parental care and proper implementation of home training for Speech and language development. ${ }^{7}$ Therefore, the current pandemic has intensified the psychological burden on families with disabilities as they cannot afford a quality consultation with SLP due to financial burden and child's communication issues. ${ }^{12,13}$

\section{Critical period of Speech and Language development}

The critical period of speech and language development is a golden period for developing effective communication skills in children. Any child failing to use this period or lack of proper stimulation can result in various future communication issues. Most of the children taking SLP services are in a critical period of speech-language development. ${ }^{14}$ They are vulnerable to facing problems like poor social integration and academic performance and fewer vocational training opportunities, affecting the overall quality of life if they fail to utilize this crucial development phase. ${ }^{7}$ Due to the outbreak of CoVID-19, children with disabilities are deprived of proper training from both SLPs \& and families, thus not utilizing the critical period. Henceforth, to prevent the adverse effect in the quality of life of children with disabilities, an alternative for SLP services is vital, as in-person service in this current situation is impossible.

\section{Telepractice Service: New Modality during Pandemic.}

With an increase in the risk of transmission in the current scenario, Tele practice can be a useful means of providing services related to audiology and speech-language therapy from a distance. ${ }^{15}$ Also, when services in person can be hindered due to geographical barriers and economic issues; this mode of service can address the problem. ${ }^{16}$ As the American Speech-Language and Hearing Association(ASHA) has assured practicing telepractice for both assessment and intervention, this can be adapted to Nepal's context.

Tele-audiology is a practice of delivering audiological services, including a comprehensive evaluation of patients at distant sites, remote hearing aid programming through an app on a smartphone, and providing clinical guidance and counseling. Although full-fledged tele-audiology practices require many important areas to consider, the patients can benefit by teaching appropriate troubleshooting of hearing aid and proper care of the devices. Different mobile applications in hearing screening also have gained interest as a possible means of hearing evaluation as a part of e-health.

\section{Care and Maintenance of Device}

1. Routine visual checks to see if any wax is present in the earmold.

2. The battery life of hearing aids may be up to 5-10 days, so it is essential to get enough batteries on shelves.

3. Minimizing the moisture content in hearing aid,

4. Cleaning the hearing aids regularly as instructed and keeping it in dry aid kit when not in use.

5. Daily listening checks need to be performed every morning before fitting the child with hearing aids. 
6. For patients requiring Mapping, they can use the initial Map and, after a month or so, using the progressive MAP or program set by the Audiologist. If they are still unable to visit the Audiologist, the patient can increase the volume units by two units every month ${ }^{3}$.

7. Monitoring progresses of the child and getting in touch with professionals about the progress.

8. The patient should get in contact with the Audiologist regularly.

The basic care and maintenance of hearing aids will help patients use the device in a time of despair maximally.

Some tips to overcome the access barrier through Telerehabilitation:

1. Parental training is crucial in this situation.

2. Tele practice and rehabilitations has its limitation; this is the only mode for accessing the service from SLPs.

3. SLPs need to be aware of the critical period about their clients, make contact with them and ensure they have access to telepractice and rehabilitation facilities and plan accordingly for therapy services.

4. Access to online sources related to rehabilitation like YouTube channels, web portals, awareness audio/video materials can be developed by collaborating with a team of SLPs and experts in the field.

5. For those who cannot avail of the teleservices, constant phone calls and counseling could be provided.

6. In the meantime, SLPs can strengthen themselves through various resources available through online access for developing their professional skills.
7. SLPs can develop an Alternative and Augmentative Communication(AAC) for those infected with COVID and are under tracheal intubation to aid in effective communication to enhance quality service in patient care. ${ }^{7}$

\section{CONCLUSIONS}

CoVID-19 has become the worst experience for humanity, even worse for those with communication disabilities. All service providers for communication therapies have become vulnerable to transmitting diseases. In this context, individuals with disabilities are deprived of availing the service from Audiologists and Speech-language pathologists, thus affecting their overall quality of life. Maintaining safety among clinicians and clients is a major concern in this present situation. However, the effect of lack of service providers in the Speech and hearing field cannot be ignored. Therefore, tele-audiology and telerehabilitation can be alternative modes of service that can be initiated for individuals with disabilities. In the present context, as ASHA has already given the emphasis on tele-practice and mentioned the practicality of it in service delivery, it is our duty as Speech and Hearing professionals to reach out to our clients and provide the service. Although few of the professionals have been giving the services through tele-mode, lack of awareness among clients and the professionals hinders its growth. For a country like Nepal where limited professionals are working in the field that too only of few major cities, the tele-mode could be beneficial for the clients from the rural areas where they are deprived of the regular services. Speech and Hearing Association of Nepal (SHAN) also should develop various awareness and training programs related to tele-practice among professionals for the client's benefit. Research on tele-practice and its efficacy can be looked into for improving the service of tele-practice in the future.

\section{REFERENCES}

1. Dong E, Du H, Gardner L. An interactive web-based dashboard to track COVID-19 in real-time. In The Lancet Infectious Diseases. 2020;20:533-4. Crossref

2. Gunjawate DR, Ravi R, Yerraguntla K, Rajashekhar B, Verma A. (2021). Impact of coronavirus disease 2019 on professional practices of audiologists and speech-language pathologists in India: A knowledge, attitude and practices survey. Clinical Epidemiology and Global Health, 2021;9:110-5.Crossref

3. Umashankar A, Prabhu P. (2020). Effect of COVID-19 on Individuals with Hearing Impairment in India. Journal of clinical and diagnostic research. 2020;14(8): MM01-MM03.Crossref

4. Scully JL. Disability, Disablism, and COVID-19 Pandemic Triage. Bioethical Inquiry.2020;17:601-5.Crossref

5. Chandrasekhar SS, Tsai Do BS, Schwartz SR, Bontempo LJ, Faucett EA, Finestone SA, et al. 2019). Clinical Practice Guideline: Sudden Hearing Loss (Update). Otolaryngology-Head and Neck Surgery. 2019;161:S1-45.Crossref

6. Woodson E, Sydlowski S. CI Surgery Cancellations due to COVID-19. In Hearing Journal. 2020;73:38-9.Crossref

7. Tohidast SA, Mansuri B, Bagheri R, Azimi H. Provision of speechlanguage pathology services for the treatment of speech and language disorders in children during the COVID-19 pandemic: Problems, concerns, and solutions. International Journal of Pediatric Otorhinolaryngology. 2020;138.110262, ISSN 0165-5876.Crossref

8. Allik H, Larsson JO, Smedje H. Health-related quality of life in parents of school-age children with Asperger syndrome or highfunctioning autism. Health and Quality of Life Outcomes, 2006;4.1. $\underline{\text { Crossref }}$

9. Aras I, Stevanović R, Vlahović S, Stevanović S, Kolarić B, Kondić L. Health related quality of life in parents of children with speech and hearing impairment. International Journal of Pediatric Otorhinolaryngology. 2014;8(2):323-9. Crossref

10. Lee MH, Matthews AK, Park C. Determinants of Health-related Quality of Life Among Mothers of Children With Cerebral Palsy. Journal of Pediatric Nursing, 2019;44:1-8.Crossref

11. Ornell F, Schuch JB, Sordi, AO, Kessler FHP. "Pandemic fear"” and COVID-19: Mental health burden and strategies. Brazilian Journal of Psychiatry. 2020;42:232-5.Crossref

12. Langevin M, Packman A, Onslow M. Parent perceptions of the impact of stuttering on their preschoolers and themselves. Journal of Communication Disorders. 2010; 43(5): 407-23.Crossref

13. Weigl V, Rudolph M, Eysholdt U, Rosanowski F. Anxiety, depression, 
and quality of life in mothers of children with cleft lip/palate. Folia Phoniatrica et Logopaedica. 2005; 57(1), 20-7. Crossref

14. Nicholas JG, Geers AE. Effects of early auditory experience on the spoken language of deaf children at 3 years of age. Ear and Hearing. 2006; 27(3):286-98. Crossref

15. Wales D, Skinner L, Hayman M. The Efficacy of Telehealth-Delivered
Speech and Language Intervention for Primary School-Age Children: A Systematic Review. International Journal of Telerehabilitation. 2017;9(1):55-70. Crossref

16. Taylor OD, Armfield NR, Dodrill P, Smith AC. A review of the efficacy and effectiveness of using telehealth for paediatric speech and language assessment. Journal of Telemedicine and Telecare. 2014;20(7):405-12. Crossref 